Pacific Journal of Mathematics

HARMONIC MAJORIZATION OF A SUBHARMONIC
FUNCTION ON A CONE OR ON A CYLINDER 


\section{HARMONIC MAJORIZATION \\ OF A SUBHARMONIC FUNCTION \\ ON A CONE OR ON A CYLINDER}

\section{H. YosHIDA}

To Professor N. Yanagihara on his 60th birthday

For a subharmonic function $u$ defined on a cone or on a cylinder which is dominated on the boundary by a certain function, we generalize the classical Phragmén-Lindelöf theorem by making a harmonic majorant of $u$ and show that if $u$ is non-negative in addition, our harmonic majorant is the least harmonic majorant. As an application, we give a result concerning the classical Dirichlet problem on a cone or on a cylinder with an unbounded function defined on the boundary.

1. Introduction. Let $\mathbb{R}$ and $\mathbb{R}_{+}$be the sets of all real numbers and all positive real numbers, respectively. The $m$-dimensional Euclidean space is denoted by $\mathbb{R}^{m}(m \geq 2)$ and $O$ denote the origin of it. By $\partial S$ and $\bar{S}$, we denote the boundary and the closure of a set $S$ in $\mathbb{R}^{m}$. Let $|P-Q|$ denote the Euclidean distance between two points $P, Q \in \mathbb{R}^{m}$. A point on $\mathbb{R}^{m}(m \geq 2)$ is represented by $(X, y), X=$ $\left(x_{1}, x_{2}, \ldots, x_{m-1}\right)$. We introduce the spherical coordinates $(r, \Theta)$, $\Theta=\left(\theta_{1}, \theta_{2}, \ldots, \theta_{m-1}\right)$, in $\mathbb{R}^{m}$ which are related to the coordinates $(X, y)$ by

$$
\left\{\begin{array}{l}
x_{1}=r\left(\prod_{j=1}^{m-1} \sin \theta_{j}\right), \quad y=r \cos \theta_{1}, \\
x_{m+1-k}=r\left(\prod_{j=1}^{k-1} \sin \theta_{j}\right) \cos \theta_{k} \quad(m \geq 3,2 \leq k \leq m-1), \\
x_{1}=r \cos \theta_{1}, \quad y=r \sin \theta_{1} \quad(m=2),
\end{array}\right.
$$

where $0 \leq r<+\infty$ and $-\frac{1}{2} \pi \leq \theta_{m-1}<\frac{3}{2} \pi \quad(m \geq 2), 0 \leq \theta_{j} \leq \pi$ $(m \geq 3,1 \leq j \leq m-2)$. The unit sphere and the surface area $2 \pi^{m / 2}\{\Gamma(m / 2)\}^{-1}$ of it are denoted by $\mathbb{S}^{m-1}$ and $s_{m}(m \geq 2)$, respectively. The upper half unit sphere $\left\{(1, \Theta) \in \mathbb{S}^{m-1} ; 0 \leq \theta_{1}<\frac{\pi}{2}\right.$ (if $m=2$, then $\left.\left.0<\theta_{1}<\pi\right)\right\}$ is also denoted by $\mathbb{S}_{+}^{m-1}(m \geq 2)$. For simplicity, a point $(1, \Theta)$ on $\mathbb{S}^{m-1}$ and a set $S, S \subset \mathbb{S}^{m-1}$, are often identified with $\Theta$ and $\{\Theta ;(1, \Theta) \in S\}$, respectively. For two 
sets $E_{1} \subset \mathbb{R}_{+}$and $E_{2} \subset \mathbb{S}^{m-1}$, the set

$$
\left\{(r, \Theta) \in \mathbb{R}^{m} ; r \in E_{1},(1, \Theta) \in E_{2}\right\}
$$

in $\mathbb{R}^{m}$ is denoted by $E_{1} \times E_{2}$. Given a domain $\Omega$ on $\mathbb{S}^{m-1} \quad(m \geq 2)$, the set $\mathbb{R}_{+} \times \Omega$ is called a cone and denoted by $C(\Omega)$. The special cone $C\left(\mathbb{S}_{+}^{m-1}\right) \quad(m \geq 2)$ called the half-space will be denoted by $\mathbb{T}_{m}$. For a positive number $r$, the set $\{r\} \times \mathbb{S}^{m-1}$ is denoted by $S_{m}(r)$ and $S_{m}(r) \cap \mathbb{T}_{m}$ by $S_{m}^{+}(r)$.

In our previous paper [12, Theorem 5.1], we gave a harmonic majorant of a certain subharmonic function $u(P)$ defined on a cone $C(\Omega)$ with a domain $\Omega$ having smooth boundary, such that

$$
\varlimsup_{P \in C(\Omega), P \rightarrow Q} u(P) \leq 0
$$

for every $Q \in \partial C(\Omega)-\{O\}$. It can be regarded as one of the generalizations of the classical Phragmén-Lindelöf theorem. We also showed in [12, Corollary 5.2] that if the function $u(P)$ is non-negative in addition, our harmonic majorant is the least harmonic majorant. In this paper, we shall consider generalizations of these results, by replacing 0 of (1.1) with a general function $g(Q)$ on $\partial C(\Omega)-\{O\}$. They were motivated by the following Theorems A, B, C and D, which are special cases of our results (see Remark 5).

Nevanlinna [10] proved

THEOREM A. Let $g(t)$ be a continuous function on $\mathbb{R}$ such that

$$
\int^{\infty} \frac{|g(t)|+|g(-t)|}{t^{2}} d t<+\infty
$$

and let $f(z)$ be a regular function on $\mathbb{T}_{2}$ such that

$$
\varlimsup_{\operatorname{Im}(z)>0, z \rightarrow t} \log |f(z)| \leq g(t)
$$

for any $t \in \partial \mathbb{T}_{2}$. If

$$
\varliminf_{r \rightarrow \infty} \frac{1}{r} \int_{0}^{\pi} \log ^{+}\left|f\left(r e^{i \theta}\right)\right| \sin \theta d \theta=0,
$$

then

$$
\log |f(z)| \leq \frac{y}{\pi} \int_{-\infty}^{+\infty} \frac{g(t)}{(t-x)^{2}+y^{2}} d t
$$

for any $z=x+i y \in \mathbb{T}_{2}$. 
In the slightly different form from Theorem A, Boas [2, pp. 92-93] also stated

THEOREM B. Make the same assumption as in Theorem A. If

$$
\varliminf_{r \rightarrow \infty} \frac{1}{r} M_{\log |f|}(r)<+\infty \quad\left(M_{\log |f|}(r)=\sup _{|z|=r, \operatorname{Im}(z)>0} \log |f(z)|\right),
$$

then

$$
\log |f(z)| \leq \frac{y}{\pi} \int_{-\infty}^{+\infty} \frac{g(t)}{(t-x)^{2}+y^{2}} d t+a_{f} y
$$

for any $z=x+i y \in \mathbb{T}_{2}$, where

$$
a_{f}=\frac{2}{\pi} \lim _{r \rightarrow \infty} \frac{1}{r} \int_{0}^{\pi} \log \left|f\left(r e^{i \theta}\right)\right| \sin \theta d \theta .
$$

Keller [7] proved an analogous result for a harmonic function on $\mathbb{T}_{3}$.

THEOREM C. Let $g(Q)$ be a continouus function on $\partial \mathbb{T}_{3}$ such that

$$
\begin{aligned}
\int^{\infty} r^{-2}\left(\int_{-\pi / 2}^{3 \pi / 2}\left|g\left(r, \frac{\pi}{2}, \theta_{2}\right)\right| d \theta_{2}\right) d r<+\infty & \\
& \left(Q=\left(r, \frac{\pi}{2}, \theta_{2}\right) \in \partial \mathbb{T}_{3}\right) .
\end{aligned}
$$

Let $h(P)$ be a harmonic function on $\mathbb{T}_{3}$ such that

$$
\varlimsup_{P \in \mathbb{T}_{3}, P \rightarrow Q} h(P) \leq g(Q)
$$

for any $Q \in \partial \mathbb{T}_{3}$.

(I) There exists

$$
b_{h^{+}}=\lim _{r \rightarrow \infty} \frac{1}{r} \int_{S_{3}^{+}(r)} h^{+}(P) \cos \theta_{1} d \sigma_{\widehat{P}}, \quad 0 \leq b_{h^{+}} \leq+\infty,
$$

where $h^{+}(P)=\max \{h(P), 0\} \quad\left(P \in S_{3}^{+}(r)\right)$ and $d \sigma_{\widehat{P}}=\sin \theta_{1} d \theta_{1} d \theta_{2}$ is the surface element on $\mathbb{S}^{2}$ at the radial projection $\widehat{P}=\left(1, \theta_{1}, \theta_{2}\right)$ of $P=\left(r, \theta_{1}, \theta_{2}\right) \in S_{3}^{+}(r)$.

(II) For any $P \in \mathbb{T}_{3}$,

$$
h(P) \leq \frac{y}{2 \pi} \int_{\partial \mathbb{T}_{3}} g(Q)|P-Q|^{-3} d Q+\frac{3}{2 \pi} b_{h^{+}} y,
$$

where $d Q$ is the area element on $\partial \mathbb{T}_{3}$. 
With respect to the least harmonic majorant of a subharmonic function on $\mathbb{T}_{m}$, Kuran [8, Theroem 3] proved

TheOREM D. Let $c<0$ and let $u(X, y)$ be subharmonic on

$$
\left\{(X, y) \in \mathbb{R}^{m} ; X \in \mathbb{R}^{m-1}, y>c\right\}
$$

such that $u \geq 0$ on $\mathbb{T}_{m}$.

(I) If

$$
\int_{\mathbb{R}^{m-1}}\left(1+|X|^{2}\right)^{-1 / 2 m} u(X, 0) d X<+\infty,
$$

then there exists the limit

$$
l_{u}=\lim _{r \rightarrow \infty} 2 m s_{m}^{-1} r^{-m-1} \int_{S_{m}^{+}(r)} y u(Q) d \sigma_{Q}, \quad 0 \leq l_{u} \leq+\infty,
$$

where $|X|=\sqrt{x_{1}^{2}+\cdots+x_{m-1}^{2}}, d X$ is the $(m-1)$-dimensional volume element at $X=\left(x_{1}, \ldots, x_{m-1}\right) \in \mathbb{R}^{m-1}(m \geq 2)$ and $d \sigma_{Q}$ is the surface element of the sphere $S_{m}(r)$ at $Q=(X, y) \in S_{m}^{+}(r)$. Further if

$$
l_{u}<+\infty
$$

then

$$
\begin{aligned}
& l_{u} y+2 s_{m}^{-1} y \int_{\mathbb{R}^{m-1}}|P-Q|^{-m} u(X, 0) d X \\
& \quad\left(P=(X, y) \in \mathbb{T}_{m}, Q=(X, 0) \in \partial \mathbb{T}_{m}\right)
\end{aligned}
$$

is the least harmonic majorant of $u(P)$ on $\mathbb{T}_{m}$.

(II) If $u$ possesses a harmonic majorant on $\mathbb{T}_{m}$, then (1.6) and (1.7) hold.

As an application, we shall give a result concerning the classical Dirichlet problem on a cone with an unbounded function defined on the boundary. Our method in this paper can be applied to a subharmonic function $u(X, y)$ defined on an infinite cylinder

$$
\left\{(X, y) \in \mathbb{R}^{m} ; X \in D, y \in \mathbb{R}\right\},
$$

where $D$ is a bounded domain in $\mathbb{R}^{m-1}(m \geq 2)$. We shall state some results in the cylindrical case.

2. Preliminaries. Let $\Lambda_{m}$ be the spherical part of the Laplace operator

$$
\Delta_{m}=\frac{\partial^{2}}{\partial x_{1}^{2}}+\frac{\partial^{2}}{\partial x_{2}^{2}}+\cdots+\frac{\partial^{2}}{\partial x_{m-1}^{2}}+\frac{\partial^{2}}{\partial y^{2}} \quad(m \geq 2)
$$


relative to the system of spherical coordinates:

$$
\Delta_{m}=\frac{m-1}{r} \frac{\partial}{\partial r}+\frac{\partial^{2}}{\partial r^{2}}+r^{-2} \Lambda_{m}
$$

Given a domain $\Omega$ on $\mathbb{S}^{m-1}$, consider the Dirichlet problem

$$
\begin{aligned}
\left(\Lambda_{m}+\lambda\right) F=0 & & \text { on } \Omega, \\
F=0 & & \text { on } \partial \Omega .
\end{aligned}
$$

We denote the least positive eigenvalue of it by $\lambda_{\Omega}^{(1)}$ and write $f_{\Omega}(\Theta)$ for the normalized positive eigenfunction corresponding to $\lambda_{\Omega}^{(1)}$, when they exist. Thus

$$
\int_{\Omega} f_{\Omega}^{2}(\Theta) d \sigma_{\Theta}=1
$$

where $d \sigma_{\Theta}$ is the surface element on $\mathbb{S}^{m-1}$. Two solutions of the equation

$$
t^{2}+(m-2) t-\lambda_{\Omega}^{(1)}=0
$$

are denoted by $\alpha_{\Omega},-\beta_{\Omega}\left(\alpha_{\Omega}, \beta_{\Omega}>0\right)$.

Let $\Phi(r, \Theta)$ be a function on $C(\Omega)$. For any given $r\left(r \in \mathbb{R}_{+}\right)$, the integral

$$
\int_{\Omega} \Phi(r, \Theta) f_{\Omega}(\Theta) d \sigma_{\Theta}
$$

is denoted by $N_{\Phi}(r)$, when it exists. The finite or infinite limits

$$
\lim _{r \rightarrow \infty} r^{-\alpha} N_{\Phi}(r) \text { and } \lim _{r \rightarrow 0} r_{\Omega} N_{\Phi}(r)
$$

are denoted by $\mu_{\Phi}$ and $\eta_{\Phi}$, respectively, when they exist. The maximum modulus $M_{\Phi}(r)(0<r<+\infty)$ of $\Phi(r, \Theta)$ is defined as

$$
M_{\Phi}(r)=\sup _{\Theta \in \Omega} \Phi(r, \Theta) \text {. }
$$

We denote $\max \{\Phi(P), 0\}$ and $\max \{-\Phi(P), 0\}$ by $\Phi^{+}(P)$ and $\Phi^{-}(P)$, respectively.

This paper is essentially based on some results in Yoshida [11]. Hence, in the subsequent consideration, we make the same assumption on $\Omega$ as in it: if $m \geq 3$, then $\Omega$ is a $C^{2, \sigma}$-domain $(0<\sigma<1)$ on $S^{m-1}$ surrounded by a finite number of mutually disjoint closed hypersurfaces (e.g., see Gilbarg and Trudinger [4, pp. 88-89] for the definition of $C^{2, \sigma}$-domain). Then there exist two positive constants $L_{1}$ and $L_{2}$ such that

$$
L_{1} \operatorname{dis}(\Theta, \partial \Omega) \leq f_{\Omega}(\Theta) \leq L_{2} \operatorname{dis}(\Theta, \partial \Omega) \quad(\Theta \in \Omega)
$$


(by modifying Miranda's method [9, pp. 7-8], we can prove this inequality).

REMARK 1. Let $\Omega=\mathbb{S}_{+}^{m-1}$. Then $\alpha_{\Omega}=1, \beta_{\Omega}=m-1$ and

$$
\begin{aligned}
f_{\Omega}(\Theta) & =\left(\begin{array}{cc}
\left(2 m s_{m}^{-1}\right)^{1 / 2} \cos \theta_{1} & (m \geq 3) \\
\frac{2}{\pi} \sin \theta & (m=2)
\end{array}\right) \\
& =\left(2 m_{m}^{-1}\right)^{1 / 2} \frac{y}{r} \quad(m \geq 2) .
\end{aligned}
$$

Let $X=\left(x_{1}, x_{2}, \ldots, x_{m-1}\right)$ be a point of $\mathbb{R}^{m-1}(m \geq 2)$. Given a bounded domain $D$ in $\mathbb{R}^{m-1} \quad(m \geq 2)$, consider the Dirichlet problem

$$
\begin{aligned}
\left(\Delta_{m-1}+\lambda\right) F & =0 & & \text { on } D, \\
F & =0 & & \text { on } \partial D .
\end{aligned}
$$

Let $\lambda_{D}$ be the least positive eigenvalue of it and let $f_{D}(X)$ be the normalized eigenfunction corresponding to $\lambda_{D}$. As in the conical case, we assume that the boundary $\partial D$ of $D \subset \mathbb{R}^{m-1} \quad(m \geq 3)$ is sufficiently smooth. The set

$$
D \times \mathbb{R}=\left\{(X, y) \in \mathbb{R}^{m} ; X \in D, y \in \mathbb{R}\right\}
$$

in $\mathbb{R}^{m}$ is called a cylinder and denoted by $\Gamma(D)(m \geq 2)$. Let $\Psi(X, y)$ be a function on $\Gamma(D)$. The integral

$$
\int_{D} \Psi(X, y) f_{D}(X) d X
$$

of $\Psi(X, y)$ is denoted by $N_{\Psi}^{\Gamma}(y)$ when it exists, where $d X$ denotes the $(m-1)$-dimensional volume element. The finite or infinite limits

$$
\lim _{y \rightarrow \infty} e^{-\sqrt{\lambda_{D}} y} N_{\Psi}(y) \text { and } \quad \lim _{y \rightarrow-\infty} e^{\sqrt{\lambda_{D}} y} N_{\Psi}(y)
$$

are denoted by $\mu_{\Psi}^{\Gamma}$ and $\eta_{\Psi}^{\Gamma}$, respectively, when they exist.

Let $G_{\Omega}(P, Q)$ (resp. $G_{D}(P, Q)$ ) be the Green function of a cone $C(\Omega)$ (resp. a cylinder $\Gamma(D)$ ) with pole at $P \in C(\Omega)$ (resp. $P \in$ $\Gamma(D)$ ), and let $\partial G_{\Omega}(P, Q) / \partial n$ (resp. $\partial G_{D}(P, Q) / \partial n$ ) be the differentiation at $Q \in \partial C(\Omega)-\{O\}$ (resp. $Q \in \partial \Gamma(D)$ ) along the inward normal into $C(\Omega)$ (resp. $\Gamma(D)$ ). It follows from our assumption on $\Omega$ (resp. $D$ ) that $\partial G_{\Omega}(P, Q) / \partial n$ (resp. $\partial G_{D}(P, Q) / \partial n$ ) is continuous on $\partial C(\Omega)-\{O\}$ (resp. $\partial \Gamma(D)$ ) (see Gilbarg and Trudinger [4, Theorem 6.15]). 
Let $g(Q)$ be a locally integrable function on $\partial C(\Omega)-\{O\}$ (resp. $\partial \Gamma(D))$ such that

$$
\begin{aligned}
& \int^{+\infty} r^{-\alpha_{\Omega}-1}\left(\int_{\partial \Omega}|g(r, \Theta)| d \sigma_{\Theta}\right) d r<+\infty, \\
& \int_{0} r^{\beta_{\Omega}-1}\left(\int_{\partial \Omega}|g(r, \Theta)| d \sigma_{\Theta}\right) d r<+\infty,
\end{aligned}
$$

(resp.

$$
\left.\int_{-\infty}^{+\infty} e^{-\sqrt{\lambda_{D}}|y|}\left(\int_{\partial D}|g(X, y)| d \sigma_{X}\right) d y<+\infty\right),
$$

where $d \sigma_{\Theta}$ (resp. $d \sigma_{X}$ ) is the surface area element of $\partial \Omega$ (resp. $\partial D$ ) at $\Theta \in \partial \Omega$ (resp. $X \in \partial D$ ). If $m=2$ and $\Omega=(\gamma, \delta)$ (resp. $D=(\gamma, \delta))$, then

$$
\begin{aligned}
& \int_{\partial \Omega}|g(r, \Theta)| d \sigma_{\Theta} \quad\left(\operatorname{resp} . \int_{\partial D}|g(X, y)| d \sigma_{X}\right) \\
&=|g(r, \gamma)|+|g(r, \delta)| \quad(\text { resp. }|g(\gamma, y)|+|g(\delta, y)|) .
\end{aligned}
$$

The Poisson integral $\mathrm{PI}_{g}(P)$ (resp. $\mathrm{PI}_{g}^{\Gamma}(P)$ ) of $g$ relative to $C(\Omega)$ (resp. $\Gamma(D))$ is defined as follows:

$$
\begin{aligned}
\mathrm{PI}_{g}(P)= & \frac{1}{c_{m}} \int_{\partial C(\Omega)-\{O\}} g(Q) \frac{\partial}{\partial n} G_{\Omega}(P, Q) d \sigma_{Q} \\
& \left(\operatorname{resp} . \mathrm{PI}_{g}^{\Gamma}(P)=\frac{1}{c_{m}} \int_{\partial \Gamma(D)} g(Q) \frac{\partial}{\partial n} G_{D}(P, Q) d \sigma_{Q}\right),
\end{aligned}
$$

where

$$
c_{m}= \begin{cases}2 \pi & (m=2), \\ (m-2) s_{m} & (m \geq 3)\end{cases}
$$

and $d \sigma_{Q}$ is the surface area element on $\partial C(\Omega)-\{O\}($ resp. $\partial \Gamma(D))$.

REMARK 2. Let $\Omega=\mathbb{S}_{+}^{m-1}$. Then

$$
G_{\Omega}(P, Q)=\left\{\begin{array}{cc}
|P-Q|^{2-m}-|P-\bar{Q}|^{2-m} & (m \geq 3), \\
-\log |P-Q|+\log |P-\bar{Q}| & (m=2),
\end{array}\right.
$$

where $\bar{Q}=(X,-y)$, that is, $\bar{Q}$ is the mirror image of $Q=(X, y)$ with respect to $\partial \mathbb{T}_{m}$. Hence, for two points $P=(X, y) \in \mathbb{T}_{m}$ and $Q \in \partial \mathbb{T}_{m}$,

$$
\frac{\partial}{\partial n} G_{\Omega}(P, Q)= \begin{cases}2(m-2)|P-Q|^{-m} y & (m \geq 3), \\ 2|P-Q|^{-2} y & (m=2) .\end{cases}
$$

3. Statement of results. The following Theorem 1 is a fundamental result in this paper. 
THEOREM 1. Let $g(Q)$ be a locally integrable function on $\partial C(\Omega)-$ $\{O\}$ satisfying (2.4) and let $u(P)$ be a subharmonic function on $C(\Omega)$ such that

$$
\varlimsup_{P \in C(\Omega), P \rightarrow Q}\left\{u(P)-\mathrm{PI}_{g}(P)\right\} \leq 0
$$

and

$$
\varlimsup_{P \in C(\Omega), P \rightarrow Q}\left\{u^{+}(P)-\mathrm{PI}_{|g|}(P)\right\} \leq 0
$$

for any $Q \in \partial C(\Omega)-\{O\}$. Then all of the limits $\mu_{u^{+}}, \eta_{u^{+}}, \mu_{u}$ and $\eta_{u}\left(0 \leq \mu_{u^{+}}, \eta_{\mu^{+}} \leq+\infty,-\infty<\mu_{u}, \eta_{u} \leq+\infty\right)$ exist, and if

$$
\mu_{u^{+}}<+\infty \text { and } \eta_{u^{+}}<+\infty \text {, }
$$

then

$$
u(P) \leq \mathrm{PI}_{g}(P)+\left(\mu_{u} r^{\alpha_{\Omega}}+\eta_{u} r^{-\beta_{\Omega}}\right) f_{\Omega}(\Theta)
$$

for any $P=(r, \Theta) \in C(\Omega)$.

REMARK 3. It is evident that (3.3) follows from

$$
\varliminf_{r \rightarrow \infty} r^{-\alpha_{\Omega}} M_{u}(r)<+\infty \text { and }{\underset{\lim }{r \rightarrow 0}}_{r} r_{\Omega} M_{u}(r)<+\infty .
$$

It is proved in Yoshida [12, Remark 9.1] that if

$$
\varlimsup_{P \in C_{m}(\Omega), P \rightarrow Q} u(P) \leq 0,
$$

for any $Q \in \partial C(\Omega)-\{O\}$, (3.5) also follows from (3.3).

REMARK 4. If $u(P)$ is a positive harmonic function on $C(\Omega)$, then (3.3) is always satisfied. To see it, apply (I) of Lemma 2 (which will be stated in $\S 4)$ to $-u(P)$. We immediately obtain that $-\infty<$ $\mu_{-u}, \eta_{-u} \leq+\infty$, so that $\mu_{u^{+}}=\mu_{u}<+\infty$ and $\eta_{u^{+}}=\eta_{u}<+\infty$.

The following Theorem 2 generalizes a result of Yoshida [11, Theorem 5].

THEOREM 2. Let $g(Q)$ be a continuous function on $\partial C(\Omega)-\{\overrightarrow{O \xi}$ satisfying (2.4) and let $u(P)$ be a subharmonic function on $C(\Omega)$ such that

$$
\varlimsup_{P \in C(\Omega), P \rightarrow Q} u(P) \leq g(Q)
$$


for any $Q \in \partial C(\Omega)-\{O\}$. Then all of the limits $\mu_{u^{+}}, \eta_{u^{+}}, \mu_{u}$ and $\eta_{u}\left(0 \leq \mu_{u^{+}}, \eta_{u^{+}} \leq+\infty,-\infty<\mu_{u}, \eta_{u} \leq+\infty\right)$ exist, and if

$$
\mu_{u^{+}}<+\infty \text { and } \eta_{u^{+}}<+\infty \text {, }
$$

then

$$
u(P) \leq \operatorname{PI}_{g}(P)+\left(\mu_{u} r^{\alpha_{\Omega}}+\eta_{u} r^{-\beta_{\Omega}}\right) f_{\Omega}(\Theta)
$$

for any $P=(r, \Theta) \in C(\Omega)$.

Corollary 1. Let $g(Q)$ be a continuous function on $\partial \mathbb{T}_{m}(m \geq 2)$ such that

$$
\int^{+\infty} r^{-2}\left(\int_{\partial \mathbb{S}_{+}^{m-1}}|g(r, \Theta)| d \sigma_{\Theta}\right) d r<+\infty .
$$

Let $u(P)$ be a subharmonic function on $\mathbb{T}_{m}$ such that

$$
\varlimsup_{P \in \mathbb{T}_{m}, P \rightarrow Q} u(P) \leq g(Q)
$$

for any $Q \in \partial \mathbb{T}_{m}$. Then both of the limits $\mu_{u^{+}}\left(0 \leq \mu_{u^{+}} \leq+\infty\right)$ and $\mu_{u}\left(-\infty<\mu_{u} \leq+\infty\right)$ exist, and

$$
u(P) \leq 2 s_{m}^{-1} \int_{\partial \mathbb{T}_{m}} g(Q)|P-Q|^{-m} d \sigma_{Q}+\left(2 m s_{m}^{-1}\right)^{1 / 2} \mu_{u^{+}} y
$$

for any $P=(X, y) \in \mathbb{T}_{m}$. If

$$
\varliminf_{r \rightarrow \infty} r^{-1} M_{u}(r)<+\infty
$$

then

$$
u(P) \leq 2 s_{m}^{-1} \int_{\partial \mathbb{T}_{m}} g(Q)|P-Q|^{-m} d \sigma_{Q}+\left(2 m s_{m}^{-1}\right)^{1 / 2} \mu_{u} y
$$

for any $P=(X, y) \in \mathbb{T}_{m}$.

REMARK 5. Let $f(z)$ be a regular function on $\mathbb{T}_{2}$. Put $m=2$ and $u(P)=\log |f(z)|$ in Corollary 1. Then (3.9) is equal to (1.2). Since (1.3) gives

$$
\mu_{\log ^{+}|f|}=0,
$$

(1.4) follows from (3.11). Since

$$
\mu_{\log |f|}=\frac{2}{\pi} \lim _{r \rightarrow \infty} \frac{1}{r} \int_{0}^{\pi} \log \left|f\left(r e^{i \theta}\right)\right| \sin \theta d \theta=\frac{\pi}{2} a_{f}
$$

(3.12) gives (1.5). Thus we obtain Theorems A and B. 
Next, to obtain Theorem C, put $m=3$ and $u=h$ in Corollary 1 . From (3.11), we have

$$
h(P) \leq \frac{y}{2 \pi} \int_{\partial \mathbb{T}_{3}} g(Q)|P-Q|^{-3} d \sigma_{\Theta}+\left(\frac{3}{2 \pi}\right)^{1 / 2} \mu_{h^{+}} y
$$

for any $P=(X, y) \in \mathbb{T}_{3}$. Since

$$
\mu_{h^{+}}=\left(\frac{3}{2 \pi}\right)^{1 / 2} b_{h^{+}}
$$

(Remark 1 with $m=3$ ), we immediately obtain Theorem C.

EXAMPLE 1. Let $\lambda_{\Omega}^{(2)}$ be the second least positive eigenvalue of (2.1) and let $F_{\Omega}(\Theta)$ be a normalized eigenfunction corresponding to $\lambda_{\Omega}^{(2)}$. Let $A_{\Omega}$ be the positive solution of the equation

$$
t^{2}+(m-2) t-\lambda_{\Omega}^{(2)}=0
$$

The harmonic function

$$
H(P)=r^{A_{\Omega}} F_{\Omega}(\Theta) \quad\left(P=(r, \Theta) \in C_{m}(\Omega)\right)
$$

on $\partial C(\Omega)$ has the property

$$
\lim _{P \in C(\Omega), P \rightarrow Q} H(P)=0,
$$

for any $Q \in \partial C(\Omega)-\{O\}$. Since $\lambda_{\Omega}^{(2)}>\lambda_{\Omega}^{(1)}$, it is evident that

$$
\lim _{r \rightarrow \infty} r^{-\alpha_{\Omega}} M_{H}(r)=+\infty \text {. }
$$

Hence it follows from Remark 3 that

$$
\mu_{H^{+}}=+\infty \text {. }
$$

This $H(P)$ shows that (3.6) with a continuous function on $\partial C(\Omega)-$ $\{O\}$ satisfying (2.4) does not always give (3.7). Further, let $g(Q)$ be a continuous function on $\partial C(\Omega)-\{O\}$ satisfying (2.4). Put

$$
I(P)=H(P)+\mathrm{PI}_{g}(P)
$$

on $C(\Omega)$. Then we see from (3.13) that $I(P)$ is a harmonic function on $C(\Omega)$ satisfying

$$
\lim _{P \in C(\Omega), P \rightarrow Q} I(P)=g(Q)
$$


for any $Q \in \partial C(\Omega)-\{O\}$ (see Lemma 3 and Lemma 6). Hence (3.6) is valid for the function $g(Q)$ on $\partial C(\Omega)-\{O\}$. However it is easy to see that $(3.8)$ is not true. Since $F_{\Omega}(\Theta)$ is orthogonal to $f_{\Omega}(\Theta)$ and

$$
N_{H}(r)=0 \quad(0<r<+\infty),
$$

it follows from Lemma 3 that

$$
\mu_{I}=\mu_{H}+\mu_{\mathrm{PI}_{g}}=0, \quad \eta_{I}=\eta_{H}+\eta_{\mathrm{PI}_{g}}=0 .
$$

Since

$$
I^{+}(P) \geq H^{+}(P)-\mathrm{PI}_{|g|}(P)
$$

on $C(\Omega)$, we see from (3.14) and Lemma 3 that

$$
\mu_{I^{+}} \geq \mu_{H^{+}}=+\infty \text {. }
$$

Hence this $I(P)$ shows that (3.8) does not always follow without (3.7).

EXAMPLE 2. There exists a subharmonic function $u(P)$ such that (3.7) is satisfied and (3.6) holds for no locally integrable function $g(Q)$ on $\partial C(\Omega)-\{O\}$ satisfying (2.4). Let $\xi$ be a number satisfying $0<$ $\xi<\frac{\pi}{2}$ and let

$$
\Omega=\left\{\Theta=\left(\theta_{1}, \theta_{2}, \ldots, \theta_{m-1}\right) \in \mathbb{S}^{m-1} ;\left|\theta_{1}\right|<\xi<\frac{\pi}{2}\right\} .
$$

Consider the subharmonic function

$$
v(r, \Theta)=r_{\Omega}^{\alpha_{\Omega}}
$$

on $C(\Omega)$ and any locally integrable function $g(Q)$ on $\partial C(\Omega)-\{O\}$ such that

$$
\varlimsup_{P \in C(\Omega), P \rightarrow Q} v(r, \Theta) \leq g(Q)
$$

at every $Q=(r, \Theta) \in \partial C(\Omega)-\{O\}$. Then we always have

$$
\int^{+\infty} r^{-\alpha_{\Omega}-1}\left(\int_{\partial \Omega}|g(r, \Theta)| d \sigma_{\Theta}\right) d r=+\infty
$$

On the other hand, we have that

$$
\lim _{r \rightarrow \infty} r^{-\alpha_{\Omega}} M_{v}(r)=1
$$

so that $\mu_{v^{+}}<+\infty$.

Let $W$ be a domain in $\mathbb{R}^{m}$ and let $g(Q)$ be a function on $\partial W$. A harmonic function on $W$ satisfying

$$
\lim _{P \in W, P \rightarrow Q} h(P)=g(Q)
$$


for any $Q \in \partial W$ is called the solution of the classical Dirichlet problem on $W$ with $g$. In comparison with a result of Keller [7, Satz in p. 25], from Theorem 2 we obtain the following Theorem 3 which gives a kind of uniqueness of solutions of the classical Dirichlet problem on an unbounded domain $C(\Omega)$. It must be remarked that the classical Dirichlet problem on unbounded domains has no unique solution (e.g. see Helms [6, p. 42 and p. 158]).

THEOREM 3. Let $g(Q)$ be a continuous function on $\partial C(\Omega)-\{O\}$ satisfying (2.4)

(I) The Poisson integral $\mathrm{PI}_{g}(P)$ is a solution of the classical Dirichlet problem on $C(\Omega)$ with $g$.

(II) Let $h(P)$ be any solution of the classical Dirichlet problem on $C(\Omega)$ with $g$. Then all of the limits $\mu_{h}, \eta_{h}\left(-\infty<\mu_{h}, \eta_{h} \leq+\infty\right)$, $\mu_{|h|}$ and $\eta_{|h|}\left(0 \leq \mu_{|h|}, \eta_{|h|} \leq+\infty\right)$ exist, and if

$$
\mu_{|h|}<+\infty \text { and } \eta_{|h|}<+\infty \text {, }
$$

then

$$
h(P)=\mathrm{PI}_{g}(P)+\left(\mu_{h} r^{\alpha_{\Omega}}+\eta_{h} r^{-\beta_{\Omega}}\right) f_{\Omega}(\Theta)
$$

for any $P=(r, \Theta) \in C(\Omega)$.

REMARK 6. The harmonic function $I(P)$ in Example 1 is one of the solutions of the classical Dirichlet problem on $C(\Omega)$, which do not satisfy (3.15). In fact, (3.14) gives

$$
\mu_{|I|}=\mu_{\left|\mathrm{PI}_{g}+H\right|}=+\infty
$$

because

$$
\mu_{\left|\mathrm{PI}_{g}\right|}=0
$$

from Lemma 3 and

$$
\mu_{\left|\mathrm{PI}_{g}+H\right|} \geq \mu_{|H|}-\mu_{\left|\mathrm{PI}_{g}\right|} \geq \mu_{H^{+}}-\mu_{\left|\mathrm{PI}_{g}\right|}=\mu_{H^{+}} .
$$

CoROLlARY 2. Let $g(Q)$ be a continuous function on $\partial C(\Omega)-\{O\}$ satisfying (2.4). If $h(P)$ is a positive harmonic function on $C(\Omega)$ which is the solution of the classical Dirichlet problem on $C(\Omega)$ with $g$, then (3.16) holds.

The following Theorem 4 generalizes a result of Yoshida [12, Corollary 5.2]. 
THEOREM 4. Let $u$ be subharmonic on a domain containing $\overline{C(\Omega)}-$ $\{O\}$ and let

$$
u \geq 0 \text { on } C(\Omega) \text {. }
$$

(I) If $\tilde{u}=u \mid \partial C(\Omega)-\{O\}$ (the restriction of $u$ to $\partial C(\Omega)-\{O\}$ ) satisfies (2.4), then both of the limits $\mu_{n}$ and $\eta_{u}\left(0 \leq \mu_{n}, \eta_{u} \leq+\infty\right)$ exist. Further, if

$$
\mu_{u}<+\infty \text { and } \eta_{u}<+\infty
$$

then

$$
h_{u}(P)=\mathrm{PI}_{\tilde{u}}(P)+\left(\mu_{u} r^{\alpha_{\Omega}}+\eta_{u} r^{-\beta_{\Omega}}\right) f_{\Omega}(\Theta) \quad(P=(r, \Theta) \in C(\Omega))
$$

is the least harmonic majorant of $u$ on $C(\Omega)$.

(II) If $u$ possesses a harmonic majorant on $C(\Omega)$, then $\tilde{u}$ satisfies (2.4) and (3.17) holds.

REMARK 7. When $u(P)$ satisfies the additional condition

$$
\lim _{P \in C(\Omega), P \rightarrow Q} u(P)=0
$$

for any $Q \in \partial C(\Omega)-\{O\}$, we extend $u(P)$ to $\mathbb{R}^{m}-\{O\}$ by defining $u(P)=0$ for any $P \in \mathbb{R}^{m}-C(\Omega)-\{O\}$. Then $u(P)$ is subharmonic on $\mathbb{R}^{m}-\{O\}$. From Remark 3 and (I) of Theorem 4, we obtain a result of Yoshida [12, Corollary 5.2].

COROLlaRY 3. Let $u$ be subharmonic on a domain containing $\overline{\mathbb{T}_{m}}$ $(m \geq 2)$ and let

$$
u \geq 0 \quad \text { on } \mathbb{T}_{m} .
$$

(I) If $\tilde{u}=u \mid \partial \mathbb{T}_{m}$ satisfies

$$
\int^{+\infty} r^{-2}\left(\int_{\partial \mathrm{s}_{+}^{m-1}} \tilde{u}(r, \Theta) d \sigma_{\Theta}\right) d r<+\infty
$$

then the limit $\mu_{u}\left(0 \leq \mu_{u} \leq+\infty\right)$ exists. Further, if

$$
\mu_{u}<+\infty
$$

then

$$
2 s_{m}^{-1} y \int_{\partial \mathbb{T}_{m}} \tilde{u}(Q)|P-Q|^{-m} d \sigma_{Q}+\left(2 m s_{m}^{-1}\right)^{1 / 2} \mu_{u} y
$$

is the least harmonic majorant of $u$ on $\mathbb{T}_{m}$. 
(II) If $u$ possesses a harmonic majorant on $\mathbb{T}_{m}$, then $\tilde{u}$ satisfies (3.18) and (3.19) holds.

REMARK 8. Theorem D immediately follows from Corollary 3. In fact, $u$ is bounded above on any compact subset of $\overline{T_{m}}$. Hence (3.19) is equivalent to (1.6). We also see from Remark 1 that

$$
l_{u}=\left(2 m s_{m}^{-1}\right)^{1 / 2} \mu_{u}
$$

and (3.20) is equal to $(1.8)$.

Finally we shall state some results in the cylindrical case.

THEOREM 5. Let $g(Q)$ be a continuous function on $\partial \Gamma(D)$ satisfying (2.5) and let $u(P)$ be a subharmonic function on $\Gamma(D)$ such that

$$
\varlimsup_{P \in \Gamma(D), P \rightarrow Q} u(P) \leq g(Q)
$$

for any $Q \in \partial \Gamma(D)$. Then all of the limits $\mu_{u^{+}}^{\Gamma} \eta_{u^{+}}^{\Gamma} \mu_{u}^{\Gamma}$ and $\eta_{u}^{\Gamma}(0 \leq$ $\left.\mu_{u^{+}}^{\Gamma}, \eta_{u^{+}}^{\Gamma} \leq+\infty,-\infty<\mu_{u}^{\Gamma}, \eta_{u}^{\Gamma} \leq+\infty\right)$ exist, and if

$$
\mu_{u^{+}}^{\Gamma}<+\infty \text { and } \eta_{u^{+}}^{\Gamma}<+\infty
$$

then

$$
u(P) \leq \mathrm{PI}_{g}(P)+\left(\mu_{u}^{\Gamma} e^{\sqrt{\lambda_{D}} y}+\eta_{u}^{\Gamma} e^{-\sqrt{\lambda_{D}} y}\right) f_{D}(X)
$$

for any $P=(X, y) \in \Gamma(D)$.

THEOREM 6. Let $g(Q)$ be a continuous function on $\partial \Gamma(D)$ satisfying (2.5).

(I) The Poisson integral $\mathrm{PI}_{g}^{\Gamma}(P)$ is a solution of the classical Dirichlet problem on $\Gamma(D)$ with $g$.

(II) Let $h(P)$ be any solution of the classical Dirichlet problem on $\Gamma(D)$ with $g$. Then all of the limits $\mu_{h}^{\Gamma}, \eta_{h}^{\Gamma}\left(-\infty<\mu_{h}^{\Gamma}, \eta_{h}^{\Gamma} \leq+\infty\right)$, $\mu_{|h|}^{\Gamma}$ and $\eta_{|h|}^{\Gamma}\left(0 \leq \mu_{|h|}^{\Gamma}, \eta_{|h|}^{\Gamma} \leq+\infty\right)$ exist, and if

$$
\mu_{|h|}^{\Gamma}<+\infty \text { and } \eta_{|h|}^{\Gamma}<+\infty
$$

then

$$
h(P)=\mathrm{PI}_{g}^{\Gamma}(P)+\left(\mu_{h}^{\Gamma} e^{\sqrt{\lambda_{D}} y}+\eta_{h}^{\Gamma} e^{-\sqrt{\lambda_{D}} y}\right) f_{D}(X)
$$

for any $P=(X, y) \in \Gamma(D)$.

COROLlARY 4. Let $g(Q)$ be a continuous function on $\partial \Gamma(D)$ satisfying (2.5). If $h(P)$ is a positive harmonic function on $\Gamma(D)$ which 
is the solution of the classical Dirichlet problem on $\Gamma(D)$ with $g$, then (3.21) holds.

THEOREM 7. Let $u$ be subharmonic on a domain containing $\overline{\Gamma(D)}$ and let

$$
u \geq 0 \text { on } \Gamma(D) \text {. }
$$

(I) If $\tilde{u}=u \mid \partial \Gamma(D)$ (the restriction of $u$ to $\partial \Gamma(D)$ ) satisfies (2.5), then both of the limits $\mu_{u}^{\Gamma}$ and $\eta_{u}^{\Gamma}\left(0 \leq \mu_{u}^{\Gamma}, \eta_{u}^{\Gamma} \leq+\infty\right)$ exist. Further, if

$$
\mu_{u}^{\Gamma}<+\infty \text { and } \eta_{u}^{\Gamma}<+\infty
$$

then

$$
\operatorname{PI}_{\tilde{u}}^{\Gamma}(P)+\left(\mu_{u}^{\Gamma} e^{\sqrt{\lambda_{D} y}}+\eta_{u}^{\Gamma} e^{-\sqrt{\lambda_{D}} y}\right) f_{D}(X) \quad(P=(X, y) \in \Gamma(D))
$$

is the least harmonic majorant of $u$ on $\Gamma(D)$.

(II) If $u$ possesses a harmonic majorant on $\Gamma(D)$, then $\tilde{u}$ satisfies (2.5) and (3.22) holds.

4. Proof of Theorem 1. For a domain $\Omega \subset \mathbb{S}^{m-1}(m \geq 2)$ and a number $t \quad(0<t<+\infty)$, the sets

$$
\begin{aligned}
& \left\{(r, \Theta) \in \mathbb{R}^{m} ; 0<r \leq t, \Theta \in \partial \Omega\right\} \quad \text { and } \\
& \left\{(r, \Theta) \in \mathbb{R}^{m} ; r \geq t, \Theta \in \partial \Omega\right\}
\end{aligned}
$$

are denoted by $S_{\Omega}^{-}(t)$ and $S_{\Omega}^{+}(t)$, respectively. For two numbers $t_{1}$ and $t_{2} \quad\left(0<t_{1}<t_{2}<+\infty\right)$, let $S_{\Omega}\left(t_{1}, t_{2}\right)$ denote the set

$$
\left\{(r, \Theta) \in \mathbb{R}^{m} ; t_{1} \leq r \leq t_{2}, \Theta \in \partial \Omega\right\} .
$$

For a point $Q \in \mathbb{R}^{m}$, the set $\left\{P \in \mathbb{R}^{m} ;|P-Q|<\rho\right\} \quad(\rho>0)$ is represented by $U_{\rho}(Q)$. We write $G_{\Omega}^{j}(P, Q)$ for the Green function of

$$
C^{j}(\Omega)=\left(j^{-1}, j\right) \times \Omega \quad(j \text { is a positive integer })
$$

with pole at $P$. For an upper semicontinuous function $\phi(Q)$ on $\partial C^{j}(\Omega)$, the Perron-Wiener-Brelot solution of the Dirichlet problem with respect to $C^{j}(\Omega)$ is denoted by $H_{\phi}^{j}(P)$ (e.g. see Helms [6]). Since the harmonic measure $\omega(P, E)$ of $E \subset \partial C^{j}(\Omega)$ with respect to $C^{j}(\Omega)$ is equal to

$$
c_{m}^{-1} \int_{E} \frac{\partial}{\partial n} G_{\Omega}^{j}(P, Q) d \sigma_{Q}
$$


(see Dahlberg [3, Theorem 3]), we know that $H_{\phi}^{j}(P)$ is equal to

$$
c_{m}^{-1} \int_{S\left(j^{-1}, j\right) \cup\left(\left\{j^{-1}\right\} \times \Omega\right) \cup(\{j\} \times \Omega)} \phi(Q) \frac{\partial}{\partial n} G_{\Omega}^{j}(P, Q) d \sigma_{Q} .
$$

To prove Theorem 1, we need some lemmas.

LEMMA 1. There exist two positive constants $k_{1}$ and $k_{2}$ (resp. $k_{3}$ and $\left.k_{4}\right)$ such that

$$
\begin{aligned}
k_{1} r^{\alpha} t^{-\beta_{\Omega}-1} f_{\Omega}(\Theta) \quad & \left(\text { resp. } k_{3} r^{-\beta_{\Omega}} t^{\alpha_{\Omega}-1} f_{\Omega}(\Theta)\right) \\
\leq \frac{\partial}{\partial n} G_{\Omega}(P, Q) \leq & k_{2} r^{\alpha_{\Omega}} t^{-\beta_{\Omega}-1} f_{\Omega}(\Theta) \\
& \left(\text { resp. } k_{4} r^{-\beta_{\Omega}} t^{\alpha_{\Omega}-1} f_{\Omega}(\Theta)\right)
\end{aligned}
$$

for $P=(r, \Theta) \in C(\Omega)$ and $Q=(t, \Phi) \in \partial C(\Omega)-\{O\}$ satisfying $0<r<\frac{1}{2} t$ (resp. $0<t<\frac{1}{2} r$ ).

Proof. These immediately follow from Azarin's inequalities [1, Lemma 1] and (2.3).

Lemma 2 (Yoshida [12, Theorem 3.31]). Let $u(P)$ be a subharmonic function on $C(\Omega)(m \geq 2)$ such that

$$
\varlimsup_{P \in C(\Omega), P \rightarrow Q} u(P) \leq 0
$$

for any $Q \in \partial C(\Omega)-\{O\}$.

(I) Both of the limits $\mu_{u}$ and $\eta_{u}\left(-\infty<\mu_{u}, \eta_{u} \leq+\infty\right)$ exist.

(II) If $\eta_{u} \leq 0$, then $r^{-\alpha_{\Omega}} N_{u}$ (r) is non-decreasing on $(0,+\infty)$.

(III) If $\mu_{u} \leq 0$, then $r^{\beta_{\Omega}} N_{u}(r)$ is non-increasing on $(0,+\infty)$.

Lemma 3. Let $g(Q)$ be a locally integrable function on $\partial C(\Omega)-\{O\}$ satisfying (2.4). Then $\mathrm{PI}_{|g|}(P)$ (resp. $\mathrm{PI}_{g}(P)$ ) is a harmonic function on $C(\Omega)$ such that both of the limits $\mu_{\mathrm{PI}_{|g|}}$ and $\eta_{\mathrm{PI}_{|g|}}$ (resp. $\mu_{\mathrm{PI}_{g}}$ and $\left.\eta_{\mathrm{PI}_{g}}\right)$ exist, and

$$
\left.\mu_{\mathrm{PI}_{|g|}}=\eta_{\mathrm{PI}_{|g|}}=0 \quad \text { (resp. } \mu_{\mathrm{PI}_{g}}=\eta_{\mathrm{PI}_{g}}=0\right) \text {. }
$$

Proof. Take any $P=(r, \Theta) \in C(\Omega)$ and two numbers $R_{1}, R_{2}$ $\left(R_{1}<\frac{1}{2} r, R_{2}>2 r\right)$. Then by Lemma 1

$$
\begin{aligned}
& c_{m}^{-1} \int_{S_{\Omega}^{+}\left(R_{2}\right)}|g(Q)| \frac{\partial}{\partial n} G_{\Omega}(P, Q) d \sigma_{Q} \\
& \quad \leq k_{5} \int_{R_{2}}^{+\infty} t^{-\alpha_{\Omega}-1}\left(\int_{\partial \Omega}|g(t, \Phi)| d \sigma_{\Phi}\right) d t,
\end{aligned}
$$


where $k_{5}=k_{2} c_{m}^{-1} r_{\Omega}^{\alpha_{\Omega}} f_{\Omega}(\Theta)$, and

$$
\begin{aligned}
& c_{m}^{-1} \int_{S_{\Omega}^{-}\left(R_{1}\right)}|g(Q)| \frac{\partial}{\partial n} G_{\Omega}(P, Q) d \sigma_{Q} \\
& \quad \leq k_{6} \int_{0}^{R_{1}} t^{\beta_{\Omega}-1}\left(\int_{\partial \Omega}|g(t, \Phi)| d \sigma_{\Phi}\right) d t
\end{aligned}
$$

where $k_{6}=k_{4} c_{m}^{-1} r^{-\beta_{\Omega}} f_{\Omega}(\Theta)$. Hence we see from (2.4) that $\mathrm{PI}_{|g|}(P)$ and $\mathrm{PI}_{g}(P)$ are finite for any $P \in C(\Omega)$. Thus $\mathrm{PI}_{g}(P)$ and $\mathrm{PI}_{|g|}(P)$ are harmonic on $C(\Omega)$.

Let $\nu_{R, P}^{(1)}(E)$ and $\nu_{R, P}^{(2)}(E) \quad(0<R<+\infty, P \in C(\Omega))$ be two positive measures on $\partial C(\Omega)-\{O\}$ such that

$$
\nu_{R, P}^{(1)}(E)=c_{m}^{-1} \int_{E \cap S_{\Omega}^{+}(R)} \frac{\partial}{\partial n} G_{\Omega}(P, Q) d \sigma_{Q}
$$

and

$$
\nu_{R, P}^{(2)}(E)=c_{m}^{-1} \int_{E \cap S_{\Omega}^{-}(R)} \frac{\partial}{\partial n} G_{\Omega}(P, Q) d \sigma_{Q}
$$

for every Borel subset $E$ of $\partial C(\Omega)-\{O\}$. Then $\mathrm{PI}_{|g|}(P)$ is the sum of two positive harmonic functions:

$$
\mathrm{PI}_{|g|}(P)=h_{1, R}(P)+h_{2, R}(P),
$$

where

$$
h_{1, R}(P)=\int_{\partial C(\Omega)-\{O\}}|g| d \nu_{R, P}^{(1)}
$$

and

$$
h_{2, R}(P)=\int_{\partial C(\Omega)-\{O\}}|g| d \nu_{R, P}^{(2)} .
$$

Let $r_{1} \quad\left(r_{1}>0\right)$ be a number and let $\varepsilon$ be any positive number. From (2.4) we can choose a number $r^{*}\left(r^{*}>2 r_{1}\right)$ so large that

$$
\int_{S_{\Omega}^{+}\left(r^{*}\right)}|g(t, \Phi)| t^{-\beta_{\Omega}-1} d \sigma_{Q} \leq \frac{c_{m}}{2 k_{2}} \varepsilon \quad(Q=(t, \Phi)) .
$$

By applying Lemma 1, we see from (4.4) that

$$
N_{h_{1, r^{*}}}\left(r_{1}\right) \leq \frac{1}{2} \varepsilon r_{1}^{\alpha_{\Omega}}
$$

and hence

$$
r_{1}^{-\alpha_{\Omega}} N_{h_{1, r^{*}}}\left(r_{1}\right) \geq-\frac{1}{2} \varepsilon .
$$

Since

$$
r^{-\alpha_{\Omega}} N_{h_{1, r^{*}}}(r)
$$


is non-decreasing from (II) of Lemma 2, (4.5) gives that

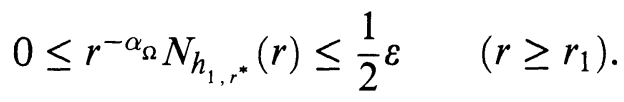

By using Lemma 1 again, we obtain that

$$
N_{h_{2, r^{*}}}(r) \leq k_{4} r^{-\beta_{\Omega}} \int_{0}^{r^{*}} t^{\beta_{\Omega}-1}\left(\int_{\partial \Omega}|g(t, \Phi)| d \Phi\right) d t \quad\left(r>2 r^{*}\right) .
$$

By (2.4) we can choose a number $r_{2}\left(r_{2}>2 r^{*}\right)$ so large that

$$
0 \leq r^{-\alpha_{\Omega}} N_{h_{2, r^{*}}}(r) \leq \frac{1}{2} \varepsilon \quad\left(r \geq r_{2}\right) .
$$

We finally conclude from (4.3), (4.6) and (4.7) that

$$
0 \leq r^{-\alpha_{\Omega}} N_{\mathrm{PI}_{|g|}}(r) \leq \varepsilon \quad\left(r \geq r_{2}\right)
$$

which gives the eixstence of $\mu_{\mathrm{PI}_{|g|}}$ and

$$
\mu_{\mathrm{PI}_{|g|}}=0 \text {. }
$$

In the same way we can also prove the existence of $\eta_{\mathrm{PI}_{|g|}}$ and

$$
\eta_{\mathrm{PI}_{|g|}}=0 \text {. }
$$

Since

$$
N_{\mathrm{PI}_{|g|}}(r) \geq N_{\left|\mathrm{PI}_{g}\right|}(r) \geq\left|N_{\mathrm{PI}_{g}}(r)\right| \quad(0<r<+\infty),
$$

it immediately follows from (4.8) and (4.9) that both limits $\mu_{\mathrm{PI}_{g}}$ and $\eta_{\mathrm{PI}_{\mathrm{g}}}$ exist and are zero.

Lemma 4 (Yoshida [12, Theorem 5.1] and Remark 3). Let $u(P)$ be a subharmonic function on $C(\Omega)(m \geq 2)$ such that

$$
\varlimsup_{P \in C(\Omega), P \rightarrow Q} u(P) \leq 0
$$

for every $Q \in \partial C(\Omega)-\{O\}$. If (3.3) is satisfied, then

$$
u(r, \Theta) \leq\left(\mu_{u} r^{\alpha_{\Omega}}+\eta_{u} r^{-\beta_{\Omega}}\right) f_{\Omega}(\Theta) \quad \text { on } C(\Omega) \text {. }
$$

Proof of Theorem 1. Consider two subharmonic functions

$$
U(P)=u(P)-\mathrm{PI}_{g}(P) \quad \text { and } \quad U^{*}(P)=u^{+}(P)-\mathrm{PI}_{|g|}(P)
$$

on $C(\Omega)$. Then we have from (3.1) and (3.2) that

$$
\varlimsup_{P \in C(\Omega), P \rightarrow Q} U(P) \leq 0 \quad \text { and } \quad \varlimsup_{P \in C(\Omega), P \rightarrow Q} U^{*}(P) \leq 0
$$


for every $Q \in \partial C(\Omega)-\{O\}$. Hence it follows from (I) of Lemma 2 that four limits $\mu_{U}, \eta_{U}, \mu_{U^{*}}$ and $\eta_{U^{*}}\left(-\infty<\mu_{U}, \eta_{U}, \mu_{U^{*}}, \eta_{U^{*}} \leq\right.$ $+\infty)$ exist. Since

$$
N_{U}(r)=N_{u}(r)-N_{\mathrm{PI}_{g}}(r) \quad \text { and } \quad N_{U^{*}}(r)=N_{u^{+}}(r)-N_{\mathrm{PI}_{|g|}}(r),
$$

Lemma 3 gives the existence of four limits $\mu_{u}, \eta_{u}, \mu_{u^{+}}$and $\eta_{u^{+}}$, and that

$$
\mu_{U}=\mu_{u}, \quad \eta_{U}=\eta_{u}, \quad \mu_{U^{*}}=\mu_{u^{+}}, \quad \eta_{U^{*}}=\eta_{u^{+}}
$$

Since

$$
U^{+}(P) \leq u^{+}(P)+\left(\mathrm{PI}_{g}\right)^{-}(P) \quad \text { on } C(\Omega),
$$

it also follows from Lemma 3 and (3.3) that

$$
\mu_{U^{+}} \leq \mu_{u^{+}}<+\infty, \quad \eta_{U^{+}} \leq \eta_{u^{+}}<+\infty .
$$

Hence by applying Lemma 4 to $U$, we can obtain from (4.10) that $U(P) \leq \mathrm{PI}_{g}(P)+\left(\mu_{u} r_{\Omega} \alpha+\eta_{u} r^{-\beta_{\Omega}}\right) f_{\Omega}(\Theta) \quad$ on $C(\Omega) \quad(P=(r, \Theta))$, which is (3.4).

5. Proofs of Theorems 2 and 3, Corollaries 1 and 2. The following lemma is not obvious for unbounded functions.

LEMMA 5. Let $g(Q)$ be an upper semicontinuous function on $\partial C(\Omega)$ $-\{O\}$ satisfying (2.4). Then

$$
\varlimsup_{P \in C(\Omega), P \rightarrow Q} \mathrm{PI}_{g}(P) \leq g(Q)
$$

for any $Q \in \partial C(\Omega)-\{O\}$,

Proof. Let $Q^{*}=\left(r^{*}, \Theta^{*}\right)$ be any point of $\partial C(\Omega)-\{O\}$ and let $\varepsilon$ be any positive number. Take a number $\delta\left(0<\delta<r^{*}\right)$. From (2.4), we can choose a number $R_{2}^{*}, R_{2}^{*}>2\left(r^{*}+\delta\right)$ (resp. $R_{1}^{*}, 0<R_{1}^{*}<$ $\left.\frac{1}{2}\left(r^{*}-\delta\right)\right)$ so large (resp. small) that

$$
\begin{gathered}
\int_{R_{2}^{*}}^{+\infty} t^{-\alpha_{\Omega}-1}\left(\int_{\partial \Omega}|g(t, \Phi)| d \sigma_{\Phi}\right) d t<\frac{c_{m}}{8 k_{2} K_{\Omega}}\left(r^{*}+\delta\right)^{-\alpha_{\Omega} \varepsilon} \\
\left(\text { resp. } \int_{0}^{R_{1}^{*}} t^{\beta_{\Omega}-1}\left(\int_{\partial \Omega}|g(t, \Phi)| d \sigma_{\Phi}\right) \mid d t<\frac{c_{m}}{8 k_{4} K_{\Omega}}\left(r^{*}-\delta\right)^{\beta_{\Omega} \varepsilon}\right),
\end{gathered}
$$

where

$$
K_{\Omega}=\max _{\Theta \in \Omega} f_{\Omega}(\Theta)
$$


From (4.1) and (4.2), we obtain that

$$
c_{m}^{-1} \int_{S_{\Omega}^{+}\left(R_{2}^{*}\right)}|g(Q)| \frac{\partial}{\partial n} G_{\Omega}(P, Q) d \sigma_{Q}<\frac{\varepsilon}{8}
$$

and

$$
c_{m}^{-1} \int_{S_{\Omega}^{-}\left(R_{1}^{*}\right)}|g(Q)| \frac{\partial}{\partial n} G_{\Omega}(P, Q) d \sigma_{Q}<\frac{\varepsilon}{8}
$$

for any $P=(r, \Theta) \in C(\Omega) \cap U_{\delta}\left(Q^{*}\right)$. Let $\varphi$ be a continuous function on $\partial C(\Omega)-\{O\}$ such that $0 \leq \varphi \leq 1$ on $\partial C(\Omega)-\{O\}$ and

$$
\varphi= \begin{cases}1 & \text { on } S_{\Omega}\left(R_{1}^{*}, R_{2}^{*}\right), \\ 0 & \text { on } S_{\Omega}^{+}\left(2 R_{2}^{*}\right) \cup S_{\Omega}^{-}\left(\frac{1}{2} R_{1}^{*}\right) .\end{cases}
$$

Since the positive harmonic function $G_{\Omega}(P, Q)-G_{\Omega}^{j}(P, Q)$ on $C^{j}(\Omega)$ converges monotonically to 0 as $j \rightarrow \infty$, we can find an integer $j_{0}$ $\left(j_{0}^{-1}<2^{-1} R_{1}^{*}, j_{0}>2 R_{2}^{*}\right)$ such that

$$
\begin{aligned}
& c_{m}^{-1} \int_{S_{\Omega}\left(2^{-1} R_{1}^{*}, 2 R_{2}^{*}\right)}|\varphi(Q) g(Q)| \\
& \quad \times\left|\frac{\partial}{\partial n} G_{\Omega}^{j_{0}}(P, Q)-\frac{\partial}{\partial n} G_{\Omega}(P, Q)\right| d \sigma_{Q}<\frac{\varepsilon}{4}
\end{aligned}
$$

for any $P=(r, \Theta) \in C(\Omega) \cap U_{\delta}\left(Q^{*}\right)$. It follows from (5.1), (5.2) and (5.3) that

$$
\begin{aligned}
&(5.4) c_{m}^{-1} \int_{\partial C(\Omega)-\{O\}} g(Q) \frac{\partial}{\partial n} G_{\Omega}(P, Q) d \sigma_{Q} \\
& \leq c_{m}^{-1} \int_{S_{\Omega}\left(2^{-1} R_{1}^{*}, 2 R_{2}^{*}\right)} \varphi(Q) g(Q) \frac{\partial}{\partial n} G_{\Omega}^{j_{0}}(P, Q) d \sigma_{Q} \\
&+\mid c_{m}^{-1} \int_{S_{\Omega}\left(2^{-1} R_{1}^{*}, 2 R_{2}^{*}\right)} \varphi(Q) g(Q) \frac{\partial}{\partial n} G_{\Omega}^{j_{0}}(P, Q) d \sigma_{Q} \\
&-c_{m}^{-1} \int_{S_{\Omega}\left(2^{-1} R_{1}^{*}, 2 R_{2}^{*}\right)} \varphi(Q) g(Q) \frac{\partial}{\partial n} G_{\Omega}(P, Q) d \sigma_{Q} \mid \\
&+2 c_{m}^{-1} \int_{S_{\Omega}^{+}\left(R_{2}^{*}\right)}|g(Q)| \frac{\partial}{\partial n} G_{\Omega}(P, Q) d \sigma_{Q} \\
&+2 c_{m}^{-1} \int_{S_{\Omega}^{-}\left(R_{1}^{*}\right)}|g(Q)| \frac{\partial}{\partial n} G_{\Omega}(P, Q) d \sigma_{Q} \\
&< c_{m}^{-1} \int_{S_{\Omega}\left(2^{-1} R_{1}^{*}, 2 R_{2}^{*}\right)} \varphi(Q) g(Q) \frac{\partial}{\partial n} G_{\Omega}^{j_{0}}(P, Q) d \sigma_{Q} \\
&+\frac{3}{4} \varepsilon
\end{aligned}
$$


for any $P=(r, \Theta) \in C(\Omega) \cap U_{\delta}\left(Q^{*}\right)$. Consider the upper semicontinuous function

$$
\begin{aligned}
& V(Q)= \begin{cases}\varphi(Q) g(Q) & \text { on } S_{\Omega}\left(2^{-1} R_{1}^{*}, 2 R_{2}^{*}\right), \\
0 & \text { on } Z\end{cases} \\
& \left(Z=S_{\Omega}\left(j_{0}^{-1}, 2^{-1} R_{1}^{*}\right) \cup S_{\Omega}\left(2 R_{2}^{*}, j_{0}\right) \cup\left(\left\{j_{0}^{-1}\right\} \times \Omega\right) \cup\left(\left\{j_{0}\right\} \times \Omega\right)\right)
\end{aligned}
$$

on $\partial C^{j_{0}}(\Omega)$. Since

$$
\varlimsup_{p \in C(\Omega), P \rightarrow Q^{*}} H_{V}^{j}(P) \leq \varlimsup_{Q \in \partial C(\Omega)-\{O\}, Q \rightarrow Q^{*}} V(Q)=g\left(Q^{*}\right)
$$

(e.g. see Helms [6, Lemma 8.20]), we finally obtain from (5.4) that

$$
\varlimsup_{P \in C(\Omega), P \rightarrow Q^{*}} \cdot c_{m}^{-1} \int_{\partial C(\Omega)-\{O\}} g(Q) \frac{\partial}{\partial n} G_{\Omega}(P, Q) d \sigma_{Q} \leq g\left(Q^{*}\right) .
$$

From Lemma 5, immediately follows

LEMMA 6. If $g(Q)$ is a continuous function on $\partial C(\Omega)-\{O\}$ satisfying (2.4), then

$$
\lim _{P \in C(\Omega), P \rightarrow Q} \mathrm{PI}_{g}(P)=g(Q)
$$

for every $Q \in \partial C(\Omega)-\{O\}$.

Proof of Theorem 2. First, we see from Lemma 6 that

$$
\lim _{P \in C(\Omega), P \rightarrow Q} \mathrm{PI}_{g}(P)=g(Q) \quad \text { and } \quad \lim _{P \in C(\Omega), P \rightarrow Q} \mathrm{PI}_{|g|}(P)=|g(Q)|
$$

for every $Q \in \partial C(\Omega)-\{O\}$. Hence we see from (3.6) that

$$
\varlimsup_{P \in C(\Omega), P \rightarrow Q}\left\{u(P)-\mathrm{PI}_{g}(P)\right\} \leq 0
$$

and

$$
\varlimsup_{P \in C(\Omega), P \rightarrow Q}\left\{u^{+}(P)-\mathrm{PI}_{|g|}(P)\right\} \leq 0
$$

for every $Q \in \partial C(\Omega)-\{O\}$. Theorem 1 immediately gives Theorem 2 .

Proof of Corollary 1. Put $\Omega=\mathbb{S}_{+}^{m-1}$ in Theorem 2. Since $g(Q)$ is continuous at $Q=O$ of $\partial \mathbb{T}_{m},|g(Q)|$ is bounded in the neighborhood of $Q=O$. Hence we see from Remark 1 and (3.9) that $g(Q)$ is admissible on $\partial \mathbb{T}_{m}$ and from (3.10) that $\eta_{u} \leq \eta_{u^{+}}=0$. If $\mu_{u^{+}}=+\infty$, then (3.11) is evidently satisfied. When $\mu_{u^{+}}<+\infty,(3.11)$ also follows 
from (3.8), Remark 1, Remark 2 and the inequality $\mu_{u} \leq \mu_{u^{+}}$. It is easily seen that Remark 3 and (3.8) give (3.12).

Proof of Theorem 3. It follows from Lemma 3 and Lemma 6 that $\mathrm{PI}_{g}(P)$ is one of the solutions. To prove (II), put $u(P)=h(P)$ and $-h(P)$ in Theorem 2 . Then Theorem 2 gives the existence of all limits $\mu_{h}, \eta_{h}, \mu_{h}^{+}, \eta_{h}^{+}$,

$$
\mu_{(-h)^{+}}=\mu_{h^{-}} \text {and } \quad \eta_{(-h)^{+}}=\eta_{h^{-}} .
$$

Since

$$
\mu_{h^{+}}+\mu_{h^{-}}=\mu_{|h|} \text { and } \eta_{h^{+}}+\eta_{h^{-}}=\eta_{\mid h^{p}},
$$

it follows that both limits $\mu_{|h|}$ and $\eta_{|h|}$ exist. Suppose that $h$ satisfies (3.15). Then we see from (5.5) and (5.6) that $\mu_{h^{+}}, \mu_{(-h)^{+}}, \eta_{h^{+}}$and $\eta_{(-h)^{+}}<+\infty$. Hence, by applying Theorem 2 to $u(P)=h(P)$ and $-h(P)$ again, we obtain from (3.8) that

$$
h(P) \leq \mathrm{PI}_{g}(P)+\left(\mu_{h} r^{\alpha_{\Omega}}+\eta_{h} r^{-\beta_{\Omega}}\right) f_{\Omega}(\Theta)
$$

and

$$
h(P) \geq \mathrm{PI}_{g}(P)+\left(\mu_{h} r^{\alpha_{\Omega}}+\eta_{h} r^{-\beta_{\Omega}}\right) f_{\Omega}(\Theta),
$$

respectively, which give (3.16).

Proof of Corollary 2. It follows from Remark 4 that

$$
\mu_{|h|}=\mu_{h^{+}}<+\infty \text { and } \eta_{|h|}=\eta_{h^{+}}<+\infty .
$$

Thus Theorem 3 implies Corollary 2.

\section{Proof of Theorem 4.}

LEMMA 7. Let $g(Q)$ be a non-negative lower semicontinuous function on $\partial C(\Omega)-\{O\}$ satisfying (2.4) and let $u(P)$ be a non-negative subharmonic function on $C(\Omega)$ such that

$$
\varlimsup_{P \in C(\Omega), P \rightarrow Q} u(P) \leq g(Q)
$$

for every $Q \in \partial C(\Omega)-\{O\}$. Then both of the limits $\mu_{u}$ and $\eta_{u}$ $\left(0 \leq \mu_{u}, \eta_{u} \leq+\infty\right)$ exist, and if $\mu_{u}<+\infty$ and $\eta_{u}<+\infty$, then

$$
u(P) \leq \mathrm{PI}_{g}(P)+\left(\mu_{u} r^{\alpha_{\Omega}}+\eta_{u} r^{-\beta_{\Omega}}\right) f_{\Omega}(\Theta)
$$

for any $P=(r, \Theta) \in C(\Omega)$. 
Proof. To apply Theorem 1, we shall show that (3.1) and (3.2) hold. Since $-g(Q)$ is upper semicontinuous on $\partial C(\Omega)-\{O\}$, it follows from Lemma 5 that

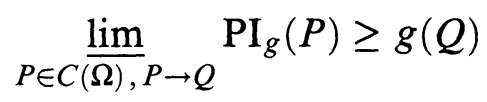

for every $Q \in \partial C(\Omega)-\{O\}$. Hence we see from (6.1) and (6.2) that

$$
\begin{aligned}
& \varlimsup_{P \in C(\Omega), P \rightarrow Q}\left\{u(P)-\mathrm{PI}_{g}(P)\right\} \\
& \quad \leq \varlimsup_{P \in C(\Omega), P \rightarrow Q} u(P)-\underbrace{\lim _{(\Omega)}}_{P \in C(\Omega), P \rightarrow Q} \operatorname{PI}_{g}(P) \leq g(Q)-g(Q)=0
\end{aligned}
$$

for every $Q \in \partial C(\Omega)-\{O\}$, which provides (3.1). Since $g$ and $u$ are non-negative, (3.2) also holds. Thus we obtain Lemma 7 from Theorem 1.

LEMMA 8. Let $u$ be subharmonic on a domain containing $\overline{C(\Omega)}-$ $\{O\}$ such that $\tilde{u}=u \mid \partial C(\Omega)-\{O\}$ satisfies (2.4) and

$$
u \geq 0 \text { on } C(\Omega) \text {. }
$$

Then

$$
\mathrm{PI}_{\tilde{u}}(P) \leq h(P) \quad \text { on } C(\Omega)
$$

for every harmonic majorant $h$ of $u$ on $C(\Omega)$.

Proof. Take any $P^{*}=\left(r^{*}, \Theta^{*}\right) \in C(\Omega)$. Let $\varepsilon$ be any positive number. In the same way as in the proof of Lemma 5 , we can choose two numbers $R_{1}$ and $R_{2} \quad\left(2 R_{1}<r<2^{-1} R_{2}\right)$ such that

$$
c_{m}^{-1} \int_{S_{\Omega}^{+}\left(R_{2}\right)} \tilde{u}(Q) \frac{\partial}{\partial n} G_{\Omega}\left(P^{*}, Q\right) d \sigma_{Q}<\frac{\varepsilon}{3}
$$

and

$$
c_{m}^{-1} \int_{S_{\Omega}^{-}\left(R_{1}\right)} \tilde{u}(Q) \frac{\partial}{\partial n} G_{\Omega}\left(P^{*}, Q\right) d \sigma_{Q}<\frac{\varepsilon}{3} .
$$

Further, take an integer $j_{0}\left(j_{0}^{-1}<R_{1}\right.$ and $\left.j_{0}>R_{2}\right)$ such that

$$
\begin{aligned}
c_{m}^{-1} \int_{S_{\Omega}\left(R_{1}, R_{2}\right)} \tilde{u}(Q)\left\{\frac{\partial}{\partial n} G_{\Omega}\left(P^{*}, Q\right)\right. & \\
& \left.-\frac{\partial}{\partial n} G_{\Omega}^{j_{0}}\left(P^{*}, Q\right)\right\} d \sigma_{Q}<\frac{\varepsilon}{3}
\end{aligned}
$$


Since

$$
c_{m}^{-1} \int_{S_{\Omega}\left(R_{1}, R_{2}\right)} \tilde{u}(Q) \frac{\partial}{\partial n} G_{\Omega}^{j_{0}}(P, Q) d \sigma_{Q} \leq H_{u}^{j_{0}}(P)
$$

for any $P \in C^{j_{0}}(\Omega)$, we have from (6.3), (6.4) and (6.5) that

$$
\begin{aligned}
& \mathrm{PI}_{\tilde{u}}\left(P^{*}\right)-H_{u}^{j_{0}}\left(P^{*}\right) \\
& \leq c_{m}^{-1} \int_{S_{\Omega}\left(R_{1}, R_{2}\right)} \tilde{u}(Q)\left\{\frac{\partial}{\partial n} G_{\Omega}\left(P^{*}, Q\right)\right. \\
& \quad+c_{m}^{-1} \int_{S_{\Omega}^{+}\left(R_{2}\right)} \tilde{u}(Q) \frac{\partial}{\partial n} G_{\Omega}\left(P^{*}, Q\right) d \sigma_{Q} \\
& \quad+c_{m}^{-1} \int_{S_{\Omega}^{-}\left(R_{1}\right)} \tilde{u}(Q) \frac{\partial}{\partial n} G_{\Omega}\left(P^{*}, Q\right) d \sigma_{Q}<\varepsilon .
\end{aligned}
$$

Here, note that $H_{u}^{j_{0}}(P)$ is the least harmonic majorant of $u(P)$ on $C^{j_{0}}(\Omega)$ (see Hayman [5, Theorem 3.15]). If $h$ is a harmonic majorant of $u$ on $C(\Omega)$, then

$$
H_{u}^{j_{0}}\left(P^{*}\right) \leq h\left(P^{*}\right)
$$

Thus we obtain from (6.6) that

$$
\mathrm{PI}_{\tilde{u}}\left(P^{*}\right)<h\left(P^{*}\right)+\varepsilon,
$$

which gives the conclusion of Lemma 8.

Proof of Theorem 4. Let $P=(r, \Theta)$ be any point of $C(\Omega)$ and let $\varepsilon$ be any positive number. By the Vitali-Carathéodory theorem (e.g. see $\left[11\right.$, p. 56]), we can find a lower semicontinuous function $g_{\varepsilon}(Q)$ on $\partial C(\Omega)-\{O\}$ such that

$$
\tilde{u}(Q) \leq g_{\varepsilon}(Q) \quad \text { on } \partial C(\Omega)-\{O\}
$$

and

$$
\mathrm{PI}_{g_{\varepsilon}}(P)<\mathrm{PI}_{\tilde{u}}(P)+\varepsilon
$$

Since

$$
\varlimsup_{P \in C(\Omega), P \rightarrow Q} u(P) \leq \tilde{u}(Q) \leq g_{\varepsilon}(Q)
$$

for any $q \in \partial C(\Omega)-\{O\}$ from (6.7), it follows from Lemma 7 that two limits $\mu_{u}, \eta_{u}$ exist and if $\mu_{u}<+\infty$ and $\eta_{u}<+\infty$, then

$$
u(P) \leq \operatorname{PI}_{g_{\varepsilon}}(P)+\left(\mu_{u} r^{\alpha_{\Omega}}+\eta_{u} r^{-\beta_{\Omega}}\right) f_{\Omega}(\Theta) .
$$


Hence we have from (6.8) and (6.9) that

$$
u(P) \leq \mathrm{PI}_{\tilde{u}}(P)+\varepsilon+\left(\mu_{u} r^{\alpha}+\eta_{u} r^{-\beta_{\Omega}}\right) f_{\Omega}(\Theta) .
$$

Since $\varepsilon$ was arbitrary, we obtain

$$
u(P) \leq \mathrm{PI}_{\tilde{u}}(P)+\left(\mu_{u} r^{\alpha_{\Omega}}+\eta_{u} r^{-\beta_{\Omega}}\right) f_{\Omega}(\Theta)
$$

for any $P=(r, \Theta) \in C(\Omega)$. This shows that $h_{u}(P)$ is a harmonic majorant of $u$ on $C(\Omega)$.

To prove that $h_{u}$ is the least harmonic majorant of $u$ on $C(\Omega)$, let $h(P)$ be any harmonic function on $C(\Omega)$ such that

$$
u(P) \leq h(P) \quad \text { on } C(\Omega) .
$$

Consider the harmonic function

$$
h^{*}(p)=h_{u}(P)-h(P) \quad \text { on } C(\Omega) .
$$

Since

$$
h^{*}(P) \leq h_{u}(P) \quad \text { on } C(\Omega),
$$

we see from Lemma 3 that $h^{*}(P)$ satisfies (3.3). We also see from Lemma 8 that

$$
\varlimsup_{P \in C(\Omega), P \rightarrow Q} h^{*}(P)=\varlimsup_{P \in C(\Omega), P \rightarrow Q}\left\{\mathrm{PI}_{\tilde{u}}(P)-h(P)\right\} \leq 0
$$

for any $Q \in \partial C(\Omega)-\{O\}$. We have from Lemma 3 and (6.10) that

$$
\mu_{h^{*}}=\mu_{h_{u}}-\mu_{h}=\mu_{u}-\mu_{h} \leq \mu_{u}-\mu_{u}=0
$$

and similarly $\eta_{h^{*}} \leq 0$. Thus we obtain from Lemma 4 that

$$
h^{*}(P) \leq 0 \quad \text { on } C(\Omega) \text {, }
$$

which shows that $h_{u}(P)$ is the least harmonic majorant of $u(P)$ on $C(\Omega)$.

To prove (II), let $h_{1}(P)$ be a harmonic majorant of $u(P)$ on $C(\Omega)$. Since

$$
\mu_{u} \leq \mu_{h_{1}}<+\infty \quad \text { and } \quad \eta_{u} \leq \eta_{h_{1}}<+\infty
$$

from Remark 4, we immediately have (3.17). Fix $P_{0}=\left(1, \Theta_{0}\right), \Theta_{0} \in$ $\Omega$. Take any two numbers $R_{1}, R_{2}\left(0<R_{1}<2^{-1}, 2<R_{2}<+\infty\right)$ and choose a sufficiently large integer $j^{*}, j^{*}>\operatorname{Max}\left(R_{1}^{-1}, R_{2}\right)$, such that

$$
c_{m}^{-1} \int_{S_{\Omega}\left(R_{1}, 2^{-1}\right)} \tilde{u}(Q)\left\{\frac{\partial}{\partial n} G_{\Omega}\left(P_{0}, Q\right)-\frac{\partial}{\partial n} G_{\Omega}^{j^{*}}\left(P_{0}, Q\right)\right\} d \sigma_{Q} \leq 1
$$


and

$$
c_{m}^{-1} \int_{S_{\Omega}\left(2, R_{2}\right)} \tilde{u}(Q)\left\{\frac{\partial}{\partial n} G_{\Omega}\left(P_{0}, Q\right)-\frac{\partial}{\partial n} G_{\Omega}^{j^{*}}\left(P_{0}, Q\right)\right\} d \sigma_{Q} \leq 1 .
$$

Since $H_{u}^{j^{*}}(P)$ is the least harmonic majorant of $u(P)$ on $C^{j^{*}}(\Omega)$,

$$
\begin{aligned}
h_{1}\left(P_{0}\right) & \geq H_{u}^{j^{*}}(P) \geq c_{m}^{-1} \int_{S_{\Omega}\left(j^{*-1}, j^{*}\right)} \tilde{u}(Q) \frac{\partial}{\partial n} G_{\Omega}^{j^{*}}\left(P_{0}, Q\right) d \sigma_{Q} \\
& \geq\left\{\begin{array}{l}
c_{m}^{-1} \int_{S_{\Omega}\left(R_{1}, 2^{-1}\right)} \tilde{u}(Q) \frac{\partial}{\partial n} G_{\Omega}^{j^{*}}\left(P_{0}, Q\right) d \sigma_{Q} \\
c_{m}^{-1} \int_{S_{\Omega}\left(2, R_{2}\right)} \tilde{u}(Q) \frac{\partial}{\partial n} G_{\Omega}^{j^{*}}\left(P_{0}, Q\right) d \sigma_{Q} .
\end{array}\right.
\end{aligned}
$$

Hence it follows from Lemma 1 that

$$
\begin{aligned}
+\infty> & h_{1}\left(P_{0}\right)+1 \\
\geq & \left\{\begin{array}{c}
c_{m}^{-1} \int_{S_{\Omega}\left(R_{1}, 2^{-1}\right)} \tilde{u}(Q) \frac{\partial}{\partial n} G_{\Omega}\left(P_{0}, Q\right) d \sigma_{Q} \\
\geq k_{1} \int_{R_{1}}^{2^{-1}} r^{-\alpha_{\Omega}-1}\left(\int_{\partial \Omega} \tilde{u}(r, \Theta) d \sigma_{\Theta}\right) d r \\
c_{m}^{-1} \int_{S_{\Omega}\left(2, R_{2}\right)} \tilde{u}(Q) \frac{\partial}{\partial n} G_{\Omega}\left(P_{0}, Q\right) d \sigma_{Q} \\
\geq k_{3} \int_{2}^{R_{2}} r_{\Omega^{-1}}\left(\int_{\partial \Omega} \tilde{u}(r, \Theta) d \sigma_{\Theta}\right) d r,
\end{array}\right.
\end{aligned}
$$

which shows that $\tilde{u}$ satisfies (2.4).

7. Proofs of Theorems 5, 6 and 7. These proofs proceed in the completely parallel way to the proofs of Theorems 2,3 and 4, on the basis of two results of Yoshida [12, Theorems 7.2 and 7.5] and the following inequality corresponding to Lemma 1 :

$$
\begin{aligned}
k_{1}^{\prime} e^{-\sqrt{\lambda_{D}}\left(y^{*}-y\right)} f_{D}(X) \quad & \left(\text { resp. } k_{3}^{\prime} e^{-\sqrt{\lambda_{D}}\left(-y^{*}+y\right)} f_{D}(X)\right) \\
\leq \frac{\partial}{\partial n} G_{D}(P, Q) \leq & k_{2}^{\prime} e^{-\sqrt{\lambda_{D}}\left(y^{*}-y\right)} f_{D}(X) \\
& \left(\text { resp. } k_{4}^{\prime} e^{-\sqrt{\lambda_{D}}\left(-y^{*}+y\right)} f_{D}(X)\right)
\end{aligned}
$$

for $P=(X, y) \in \Gamma(D)$ and $Q=\left(X^{*}, y^{*}\right) \in \partial \Gamma(D)$ satisfying $y^{*}>$ $y+1$ (resp. $y^{*}<y-1$ ), where $k_{1}^{\prime}$ and $k_{2}^{\prime}$ (resp. $k_{3}^{\prime}$ and $k_{4}^{\prime}$ ) are two positive constants.

\section{REFERENCES}

[1] V. S. Azarin, Generalization of a theorem of Hayman on subharmonic functions in an m-dimensional cone, Mat. Sb., 66 (108) (1965), 248-264; English transl. in Amer. Math. Soc. Transl. (2) 80 (1969), 119-138.

[2] R. P. Boas, Entire Functions, Academic, New York, 1954. 
[3] B. E. J. Dahlberg, Estimates of harmonic measure, Arch. Rational Mech. Anal., 65 (1977), 275-288.

[4] D. Gilbarg and N. S. Trudinger, Elliptic Partial Differential Equations of the Second Order, Springer, Berlin, 1977.

[5] W. K. Hayman and P. W. Kennedy, Subharmonic Functions, vol. 1, Academic, London, 1976.

[6] L. L. Helms, Introduction to Potential Theory, Wiley-Interscience, New York, 1969.

[7] H. Keller, Über das Anwachsen von Potentialfunktionen im dreidimensionalen Raum, Ann. Acad. Sci. Fenn. Ser. A, 83 (1950), 35 pp.

[8] U. Kuran, Harmonic majorizations in half-balls and half-spaces, Proc. London Math. Soc., (3) 21 (1970), 614-636.

[9] C. Miranda, Partial Differential Equations of Elliptic Type, Springer, Berlin, 1970.

[10] R. Nevanlinna, Über die Eigenschaften meromorpher Funktionen in einem Winkelraum, Acta Soc. Sci. Fenn., 50 No. 12 (1922), 45 pp.

[11] W. Rudin, Real and Complex Analysis, Third Edition, McGraw-Hill, New York, 1986.

[12] H. Yoshida, Nevanlinna norm of a subharmonic function on a cone or on a cylinder, Proc. London Math. Soc., (3) 54 (1987), 267-299.

Received October 26, 1988, and in revised form October 31, 1989.

CHIBA UNIVERSITY

1-33 YAYOI-CHO

CHIBA-CITY 260, JAPAN 



\section{PACIFIC JOURNAL OF MATHEMATICS EDITORS}

\author{
V. S. VARADARAJAN \\ (Managing Editor) \\ University of California \\ Los Angeles, CA 90024-1555-05 \\ Herbert Clemens \\ University of Utah \\ Salt Lake City, UT 84112 \\ THOMAS ENRIGHT \\ University of California, San Diego \\ La Jolla, CA 92093
}

R. FINN

Stanford University

Stanford, CA 94305

HeRmann FlaschKa

University of Arizona

Tucson, AZ 85721

VAUghan F. R. Jones

University of California

Berkeley, CA 94720

SteVEN KeRCKHOFF

Stanford University

Stanford, CA 94305

\section{C. MOORE}

University of California

Berkeley, CA 94720

MARTIN SCHARLEMANN

University of California

Santa Barbara, CA 93106

HAROLD STARK

University of California, San Diego

La Jolla, CA 92093

\section{ASSOCIATE EDITORS \\ R. ARENS \\ E. F. BECKENBACH \\ B. H. NeUmanN \\ F. WoLF \\ (1904-1989) \\ K. Yoshida (1906-1982)}

\section{SUPPORTING INSTITUTIONS}

UNIVERSITY OF ARIZONA

UNIVERSITY OF BRITISH COLUMBIA

CALIFORNIA INSTITUTE OF TECHNOLOGY

UNIVERSITY OF CALIFORNIA

MONTANA STATE UNIVERSITY

UNIVERSITY OF NEVADA, RENO

NEW MEXICO STATE UNIVERSITY

OREGON STATE UNIVERSITY
UNIVERSITY OF OREGON

UNIVERSITY OF SOUTHERN CALIFORNIA

STANFORD UNIVERSITY

UNIVERSITY OF HAWAII

UNIVERSITY OF TOKYO

UNIVERSITY OF UTAH

WASHINGTON STATE UNIVERSITY

UNIVERSITY OF WASHINGTON

The Supporting Institutions listed above contribute to the cost of publication of this Journal, but they are not owners or publishers and have no responsibility for its content or policies.

Mathematical papers intended for publication in the Pacific Journal of Mathematics should be in typed form or offset-reproduced (not dittoed), double spaced with large margins. Please do not use built up fractions in the text of the manuscript. However, you may use them in the displayed equations. Underline Greek letters in red, German in green, and script in blue. The first paragraph must be capable of being used separately as a synopsis of the entire paper. In particular it should contain no bibliographic references. Please propose a heading for the odd numbered pages of less than 35 characters. Manuscripts, in triplicate, may be sent to any one of the editors. Please classify according to the 1991 Mathematics Subject Classification scheme which can be found in the December index volumes of Mathematical Reviews. Supply name and address of author to whom proofs should be sent. All other communications should be addressed to the managing editor, or Elaine Barth, University of California, Los Angeles, California 90024-1555-05.

There are page-charges associated with articles appearing in the Pacific Journal of Mathematics. These charges are expected to be paid by the author's University, Government Agency or Company. If the author or authors do not have access to such Institutional support these charges are waived. Single authors will receive 50 free reprints; joint authors will receive a total of 100 free reprints. Additional copies may be obtained at cost in multiples of 50 .

The Pacific Journal of Mathematics (ISSN 0030-8730) is published monthly except for July and August. Regular subscription rate: $\$ 190.00$ a year (10 issues). Special rate: $\$ 95.00$ a year to individual members of supporting institutions.

Subscriptions, orders for numbers issued in the last three calendar years, and changes of address should be sent to Pacific Journal of Mathematics, P.O. Box 969, Carmel Valley, CA 93924, U.S.A. Old back numbers obtainable from Kraus Periodicals Co., Route 100, Millwood, NY 10546.

The Pacific Journal of Mathematics at P.O. Box 969, Carmel Valley, CA 93924 (ISSN 0030-8730) is published monthly except for July and August. Second-class postage paid at Carmel Valley, California 93924, and additional mailing offices. Postmaster: send address changes to Pacific Journal of Mathématics, P.O. Box 969, Carmel Valley, CA 93924.

PUBLISHED BY PACIFIC JOURNAL OF MATHEMATICS, A NON-PROFIT CORPORATION Copyright (C) 1991 by Pacific Journal of Mathematics 


\section{Pacific Journal of Mathematics}

Vol. 148, No. $2 \quad$ April, 1991

Yuri A. Abramovich, Operators preserving disjointness on rearrangement

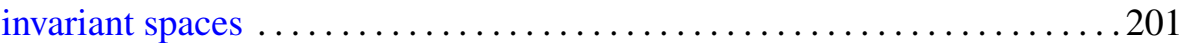

Andrew French Acker and Kirk Lancaster, Existence and geometry of a free boundary problem for the heat equation .................. 207

So-Chin Chen, Real analytic regularity of the Szegő projection on circular

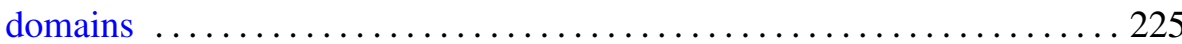

Chen-Lian Chuang, An independence property of central polynomials . . . 237

Peter Larkin Duren and M. Schiffer, Robin functions and energy

functionals of multiply connected domains $\ldots \ldots \ldots \ldots \ldots \ldots \ldots \ldots . \ldots 251$

Johan Henricus Bernardus Kemperman, Sets of uniqueness and systems

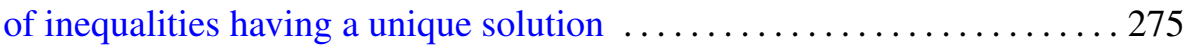

Ka-Lam Kueh, Fourier coefficients of nonholomorphic modular forms and sums of Kloosterman sums . .......................... 303

Gerard J. Murphy, Ordered groups and crossed products of $C^{*}$-algebras . . 319

You-Qiang Wang, The $p$-parts of Brauer character degrees in $p$-solvable

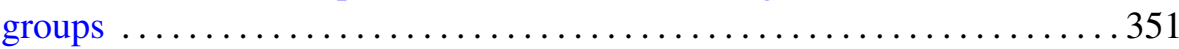

Hidenobu Yoshida, Harmonic majorization of a subharmonic function on a cone or on a cylinder ........................................ 369 